DOI: https://doi.org/10.24297/jam.v17i0.8528

\title{
On Three Dimensional Pseudosymmetric Alpha-Kenmotsu Manifolds
}

\author{
Hakan Öztürk ${ }^{1}$ and Sunil Kumar Yadav ${ }^{2}$ \\ ${ }^{1}$ Afyon Kocatepe University, Afyon Vocational School, Campus of ANS, Afyonkarahisar, Turkey \\ ${ }^{2}$ Department of Mathematics, Poornima College of Engineering, ISI-6, RIICO Institutional Area, Sitapura, \\ Jaipur- 302022, Rajasthan, India \\ hozturk@aku.edu.tr, prof_sky16@yahoo.com
}

\section{Abstract}

The main purpose of this paper is to study pseudosymmetric conditions on alpha-Kenmotsu manifolds with dimension 3. In particular, we obtain some results satisfying some certain curvature conditions on such manifolds depending on $\alpha$.

Keywords: Kenmotsu manifold, Alpha-Kenmotsu manifold, Pseudosymmetry, Einstein manifold.

\section{Introduction}

Let $(M, g)$ be an $n$-dimensional $(n \geq 3)$ differentiable manifold of class $C^{\infty}$. Denote by $\nabla$ its Levi-Civita connection. Also, we define endomorphisms $R(X, Y)$ and $(X \wedge Y)$ by the following relations:

$$
R(X, Y) Z=\nabla_{X} \nabla_{Y} Z-\nabla_{Y} \nabla_{X} Z-\nabla_{[X, Y]} Z
$$

and

$$
(X \wedge Y) Z=g(Y, Z) X-g(Z, X) Y,
$$

respectively, [16]. Here $X, Y, Z \in \chi(M), \chi(M)$ being the Lie algebra of vector fields on $M$. The Riemannian Christoffel curvature tensor $R$ is defined as

$$
g(R(X, Y) V, W)=g(R(X, Y) V, W)
$$

where $W \in \chi(M)$. We define the tensors $R . R$ and $Q(g, R)$ by the following relations:

$$
\begin{gathered}
(R(X, Y) \cdot R)(U, W) Z=R(X, Y) R(U, W) Z-R(R(X, Y) U, W) Z \\
-R(U, R(X, Y) W) Z-R(U, W) R(X, Y) Z
\end{gathered}
$$

and

$$
\begin{aligned}
Q(g, R)(U, W, Z ; X, Y) & =(X \wedge Y) R(U, W) Z-R((X \wedge Y) U, W) Z \\
& -R(U,(X \wedge Y) W) Z-R(U, W)(X \wedge Y) Z
\end{aligned}
$$

where $X, Y, Z, U, W \in \chi(M),[3]$.

If the tensors $R . R$ and $Q(g, R)$ are linearly dependent then the manifold $M$ is said to be pseudosymmetric. This condition is equivalent to

$$
R \cdot R=L_{R} Q(g, R)
$$


holding on the set

$$
U_{R}=\{x \in M: Q(g, R) \neq 0 \text {, at the point } x\},
$$

where $L_{R}$ is some function on $U_{R^{\prime}}$ [3].

If $R . R=0$ then the manifold $M$ is called semi symmetric. We know that every semi symmetric manifold is pseudosymmetric. But the converse is not true. Moreover, if $\nabla R=0$ then the manifold $M$ is said to be locally symmetric. Also, it is obvious that if the manifold $M$ is locally symmetric then it is semi symmetric [14].

The notion of a semi-symmetric manifold is defined by $R(X, Y) \cdot R=0$, for all vector fields $X, Y$ on $M$, where $R(X, Y)$ acts as a derivation on $R$, [9]. Such a space is called "semi-symmetric space" since the curvature tensor of $(M, g)$ at a point $p \in M, R p$; is the same as the curvature tensor of a symmetric space (that can change with the point of p). Thus locally symmetric spaces are obviously semi-symmetric, but the converse is not true, [4]. Complete intrinsic classification of these spaces was given by Szabó, [14]. However, it is interesting to investigate the semi-symmetry of special Riemannian manifolds. Nomizu proved that if $M^{\mathrm{n}}$ is a complete, connected semisymmetric hypersurfaces of a Euclidean space $R^{\mathrm{n}+1}, n>3$, i.e., $R \cdot R=0$, then $M^{\mathrm{n}}$ is locally symmetric, i.e., $\nabla R=$ 0 . For the case of a compact Kaehlerian manifold, Ogawa proved that if it is semi-symmetric then it must be locally symmetric, [10].

Semisymmetric Kenmotsu and alpha-Kenmotsu manifolds are studied in $[8,13]$. Also, the other semi symmetric conditions are investigated in $[11,15]$ on such manifolds. In this paper, we study pseudosymmetric conditions on alpha-Kenmotsu manifolds with dimension 3. In particular, we obtain some results satisfying some certain curvature conditions on such manifolds depending on $\alpha$.

\section{Alpha-Kenmotsu Manifolds}

Let $M^{2 \mathrm{n}+1}$ almost contact manifold be an odd-dimensional manifold. The triple $(\varphi, \xi, \eta)$ is defined as follow. It transports a field $\varphi$ of endomorphisms of the tangent spaces, $\xi$ is a vector field that is called characteristic or Reeb vector field and $\eta$ is a 1 -form such that $\varphi^{2}=-I+\eta \otimes \xi$ and $\eta(\xi)=1$. The mapping defined by $I: T M^{2 \mathrm{n}+1} \rightarrow T M^{2 \mathrm{n}+1}$ is called identity mapping. By using the definition of these it follows that $\varphi \xi=0, \eta \circ \varphi=0$ and that the $(1,1)$-tensor field $\varphi$ has constant rank $2 n$ [16]. An almost contact manifold $\left(M^{2 n+1}, \varphi, \xi, \eta\right)$ is said to be normal if the Nijenhuis torsion tensor of $\varphi ; N=[\varphi, \varphi]+2 d \eta \otimes \xi$ vanishes for any vector fields $X, Y$ on $M^{2 \mathrm{n}+1}$. If $M^{2 \mathrm{n}+1}$ admits a Riemannian metric $g$, such that

$$
g(\varphi X, \varphi Y)=g(X, Y)-\eta(X) \eta(Y),
$$

for any vector fields $X, Y$ on $M^{2 \mathrm{n}+1}$, then this metric $g$ is said to be a compatible metric and the manifold $M^{2 \mathrm{n}+1}$ together with the structure $\left(M^{2 \mathrm{n}+1}, \varphi, \xi, \eta, g\right)$ is called an almost contact metric manifold. Hence, (2.1) means that $\eta(X)=g(X, \xi)$ for any vector field $X$ on $M^{2 \mathrm{n}+1}$. On such a manifold, the fundamental 2-form $\Phi$ of $M^{2 \mathrm{n}+1}$ is defined by $\Phi(X, Y)=g(\varphi X, Y)$. Almost contact metric manifolds such that both $\eta$ and $\Phi$ are closed are called almost symplectic manifolds and almost contact metric manifolds such that $d \eta=0$ and $d \Phi=2 \eta \wedge \Phi$ are almost Kenmotsu manifolds. It is noted that a normal almost symplectic manifold is called a symplectic manifold and a normal almost Kenmotsu manifolds is called Kenmotsu manifold [6].

An almost contact metric manifold $M^{2 \mathrm{n}+1}$ is said to be almost alpha-Kenmotsu if $d \eta=0$ and $d \Phi=2 \alpha \eta \wedge \Phi, \alpha$ being a non-zero real constant [6].

Now, we set $A=-\nabla \xi$ and $h=(1 / 2)\left(L_{\xi} \varphi\right)$. Obviously, $A(\xi)=0$ and $h(\xi)=0$. Moreover, the tensor fields $A$ and $h$ are symmetric operators and satisfy the following relations 
$\nabla_{X} \xi=-\alpha \varphi^{2} X-\varphi h X$

$(\varphi \circ h) X+(h \circ \varphi) X=0$,

$(\varphi \circ A) X+(A \circ \varphi) X=-2 \alpha \varphi$,

$\left(\nabla_{X} \eta\right) Y=\alpha[g(X, Y)-\eta(X) \eta(Y)]+g(\varphi Y, h X)$,

$\delta \eta=-2 \alpha n, \operatorname{tr}(h)=0$,

for any vector fields $X, Y$ on $M^{2 \mathrm{n}+1}$. We also remark that $h=0 \Leftrightarrow \nabla \xi=-\alpha \varphi^{2}[12,13]$

For an almost alpha-Kenmotsu manifold, the following curvature properties are held:

$$
\begin{gathered}
R(X, Y) \xi=\left(\nabla_{Y} \varphi h\right) X-\left(\nabla_{X} \varphi h\right) Y-\alpha[\eta(X) \varphi h Y-\eta(Y) \varphi h X](Y \\
+\left[\alpha^{2}+\xi(\alpha)\right][\eta(X) Y-\eta(Y) X] \\
R(X, \xi) \xi=\left[\alpha^{2}+\xi(\alpha)\right] \varphi^{2} X+2 \alpha \varphi h X-h^{2} X+\varphi\left(\nabla_{\xi} h\right) X \\
\left(\nabla_{\xi} h\right) X=-\varphi R(X, \xi) \xi-\left[\alpha^{2}+\xi(\alpha)\right] \varphi X-2 \alpha h X-\varphi h^{2} X \\
R(X, \xi) \xi-\varphi R(\varphi X, \xi) \xi=2\left[\left(\alpha^{2}+\xi(\alpha)\right) \varphi^{2} X-h^{2} X\right] \\
S(X, \xi)=-2 n\left[\alpha^{2}+\xi(\alpha)\right] \eta(X)-(\operatorname{div}(\varphi h)) X \\
S(\xi, \xi)=-\left[2 n\left(\alpha^{2}+\xi(\alpha)\right)+\operatorname{tr}\left(h^{2}\right)\right]
\end{gathered}
$$

for any vector fields $X, Y$ on $M^{2 \mathrm{n}+1}$ where $\alpha$ is a smooth function such that $d \alpha \wedge \eta=0$. In these formulas, $\nabla$ is the Levi-Civita connection and $R$ the Riemannian curvature tensor of $M^{2 n+1}[12,13,15]$.

\section{Results and Discussion}

In this section, we consider pseudosymmetric alpha-Kenmotsu manifolds with dimension 3 . Here, $\alpha$ is a smooth function such that $d \alpha \wedge \eta=0$ or a real constant.

Proposition 3.1 Let $M^{n}$ be an alpha-Kenmotsu manifold. Then the following equations are held:

$$
\begin{gathered}
\left(\nabla_{X} \varphi\right) Y=-\alpha[g(X, \varphi Y) \xi+\eta(Y) \varphi X] \\
\nabla_{X} \xi=-\alpha(-X+\eta(X) \xi),\left(\nabla_{X} \eta\right) Y=-\alpha[-g(X, Y)+\eta(X) \eta(Y)],
\end{gathered}
$$

where $\alpha$ is strictly positive function of class $C^{\infty}$ such that $d \alpha \wedge \eta=0$. In special cases, if $\alpha=0$, then the manifold is a symplectic one. Also, if $\xi(\alpha)=\nabla_{\xi} \alpha$ such that $\left(\alpha^{2}+\xi(\alpha)\right) \neq 0$, then the alpha-Kenmotsu manifold is regular [6]. It is important to say that the condition $d \alpha \wedge \eta=0$ satisfies for dimension is greater and equal than 5 . This condition does not hold for the three dimensional case [15]. Accordingly, since the conformal curvature tensor in the three dimensional space will be identical to zero, we can also make the Riemannian curvature tensor calculations on the conformal curvature tensor. In other words, we have

$$
\begin{aligned}
R(X, Y) Z=S(Y, Z) X- & S(Z, X) Y+g(Y, Z) Q X \\
& \quad-g(Z, X) Q Y-(r / 2)[(X \wedge Y) Z] .
\end{aligned}
$$


So we can give the following results for the three dimensional case:

Proposition 3.2 In three dimensional alpha-Kenmotsu manifolds, we have the following relations:

$$
\begin{gathered}
R(X, Y) Z=2\left(\alpha^{2}+\xi(\alpha)+(r / 4)\right)((X \wedge Y) Z) \\
-3\left(\alpha^{2}+\xi(\alpha)+(r / 6)\right)[\eta(X)(\xi \wedge Y) Z+\eta(Y)(X \wedge \xi) Z] \\
S(X, Y) Z=\left(\alpha^{2}+\xi(\alpha)+(r / 2)\right) g(X, Y)-3\left(\alpha^{2}+\xi(\alpha)+(r / 6)\right) \eta(X) \eta(Y),
\end{gathered}
$$

where $\alpha$ is a strictly positive function such that $d \alpha \wedge \eta=0$. Moreover, $R, S, Q$ and $r$ are the Riemannian curvature tensor, the Ricci tensor, the Ricci operator and the scalar curvature, respectively [15].

Proposition 3.3 In three dimensional alpha-Kenmotsu manifolds, taking into account of (3.3), (3.4) and (3.5), the following curvature relations are held:

$$
\begin{aligned}
& R(X, Y) \xi=\left(\alpha^{2}+\xi(\alpha)\right)[\eta(X) Y-\eta(Y) X] \\
& R(\xi, X) Y=\left(\alpha^{2}+\xi(\alpha)\right)[-g(X, Y) \xi+\eta(Y) X] \\
& g(R(X, Y) Z, \xi)=\left(\alpha^{2}+\xi(\alpha)\right)[g(X, Z) \eta(Y)-g(Y, Z) \eta(X)], \\
& S(Y, \xi)=-2\left(\alpha^{2}+\xi(\alpha)\right) \eta(Y), \\
& Q \xi=-2\left(\alpha^{2}+\xi(\alpha)\right) \xi
\end{aligned}
$$

$[12,13]$

Theorem 3.1 Let $M^{3}$ be an alpha-Kenmotsu manifold. If $M^{3}$ is pseudosymmetric then either it is locally isometric to the hyperbolic space $H^{3}\left(-\alpha^{2}\right)$ or $L_{R}=-\alpha^{2}$ holds where $\alpha$ is a real constant.

Proof Suppose that $M^{3}$ is an alpha-Kenmotsu manifold and $\alpha$ is a real constant. If $M^{3}$ is semi symmetric then it is clearly pseudosymmetric. Also, a semi symmetric alpha-Kenmotsu manifold is locally isometric to the hyperbolic space $H^{3}\left(-\alpha^{2}\right)$ [8]. In this case, let us assume that $M^{3}$ is not semi symmetric. In other words, let $M^{3}$ be a pseudosymmetric alpha-Kenmotsu manifold. In view of (1.1) and (3.7) we have

$$
R(\xi, X) Y=\alpha^{2}(X \wedge \xi) Z
$$

Now, we consider the pseudosymmetry condition

$R(X, Y) \cdot R=L_{R}[(X \wedge Y) \cdot R]$.

So we get

$$
(R(X, Y) \cdot R)(U, V) Z=L_{R} Q(g, R)(U, V, Z ; X, Y)
$$

for arbitrary vector fields on $M^{3}$. Here the tensor product is defined as

$$
R(X, Y) R(U, V) Z-R(R(X, Y) U, V) Z-R(U, R(X, Y) V) Z-R(U, V) R(X, Y) Z
$$


$=L_{R}[(X \wedge Y) R(U, V) Z-R((X \wedge Y) U, V) Z-R(U,(X \wedge Y) V) Z-R(U, V)(X \wedge Y) Z]$.

With the help of (3.7) taking $X=\xi$ in (3.13), we have

$$
R(\xi, X) \cdot R=\alpha^{2}[(X \wedge \xi) \cdot R]
$$

This implies that $L_{R}=-\alpha^{2}$. Thus the poof is completed.

Theorem 3.2 Let $M^{3}$ be an alpha-Kenmotsu manifold. If $M^{3}$ is pseudosymmetric then either it is locally isometric to the hyperbolic space $H^{3}\left(-\alpha^{2}\right)$ or $L_{R}=\left(-\alpha^{2}+\xi(\alpha)\right)$ holds where $\alpha$ is strictly positive function of class $C^{\infty}$ such that $d \alpha \wedge \eta=0$.

Proof Firstly we assume that $M^{3}$ is semi-symmetric, then the manifold is also pseudosymmetric and in [8], it is clear that a semi symmetric alpha-Kenmotsu manifold is locally isometric to the hyperbolic space $H^{3}\left(-\alpha^{2}\right)$. Conversely, we suppose that $M^{3}$ is not a semi symmetric alpha-Kenmotsu manifold. By using the methodology of the above theorem and considering (1.1), (3.7) and (3.13), we obtain

$$
\begin{aligned}
& 0=\left[L_{R}+\left(\alpha^{2}+\xi(\alpha)\right)\right]\left[R(U, V, Z, Y)-\left(\alpha^{2}+\xi(\alpha)\right)[-g(V, Z) \eta(U) \eta(Y)\right. \\
& +g(U, Z) \eta(V) \eta(Y)-g(U, Y) g(V, Z)+g(U, Y) \eta(V) \eta(Z)+g(V, Z) \eta(Y) \eta(U) \\
& -g(Y, Z) \eta(V) \eta(U)-g(V, Y) \eta(U) \eta(Z)+g(V, Y) g(Z, U)+g(Y, Z) \eta(U) \eta(V) \\
& -g(Z, U) \eta(V) \eta(Y)+g(Y, V) \eta(U) \eta(Z)-g(Y, U) \eta(Z) \eta(V)]] .
\end{aligned}
$$

Let $\left\{e_{i}\right\}, i=1,2,3$ be an orthonormal basis of the tangent space at any point. Then the sum for $1 \leq i \leq 3$ of (3.15) for suitable contraction yields

$$
0=\left[L_{R}+\left(\alpha^{2}+\xi(\alpha)\right)\right]\left(S(V, Z)+2\left(\alpha^{2}+\xi(\alpha)\right) g(V, Z)\right)
$$

for $U=Y=e_{i}, i=1,2,3$. For $Z=\xi$, the last equation leads to

$$
0=\left[L_{R}+\left(\alpha^{2}+\xi(\alpha)\right)\right]
$$

This proves the theorem.

Corollary 3.1 Every alpha-Kenmotsu manifold $M^{3}$ is a pseudosymmetric manifold of the form

$$
R \cdot R=-\left(\alpha^{2}+\xi(\alpha)\right) Q(g, R)
$$

where $\alpha$ is strictly positive function of class $C^{\infty}$ such that $d \alpha \wedge \eta=0$. For $\left.\xi(\alpha)\right)=0$, we have

$$
R \cdot R=-\alpha^{2} Q(g, R)
$$

Theorem 3.3 Let $M^{3}$ be a pseudosymmetric alpha-Kenmotsu manifold. If the manifold $M^{3}$ has a non-zero function such that $-L_{R}=\left(\alpha^{2}+\xi(\alpha)\right)$ then the manifold $M^{3}$ is an Einstein manifold with $\lambda=-2\left(\alpha^{2}+\xi(\alpha)\right)$ where $\alpha$ is strictly positive function of class $C^{\infty}$ such that $d \alpha \wedge \eta=0$.

Proof Assume that $M^{3}$ is a pseudosymmetric alpha-Kenmotsu manifold. Thus we have $R \cdot R=L_{R} Q(g, R)(U, V, Z ; X, Y)$. 
Next, taking into account of the last equation, (1.1), (3.13) and $X=\xi$, we get

$$
\begin{gathered}
0=\left[L_{R}+\left(\alpha^{2}+\xi(\alpha)\right)\right][g(R(U, V) Z, Y) \xi-g(R(U, V) Z), \xi Y \\
-g(U, Y) R(\xi, V) Z+\eta(U) R(Y, V) Z-g(V, Y) R(U, \xi) Z \\
+\eta(V) R(U, Y) Z-g(Z, Y) R(U, V) \xi+\eta(Z) R(U, V) Y] .
\end{gathered}
$$

Then taking inner product both two sides with respect to $\xi$, we obtain

$$
\begin{aligned}
0=\left[L_{R}+\left(\alpha^{2}+\xi(\alpha)\right)\right][ & R(U, V) Z, Y-\eta(Y) g(R(U, V) Z, \xi) \\
& -g(U, Y) g(R(\xi, V) Z, \xi)+\eta(U) g(R(Y, V) Z, \xi) \\
& -g(V, Y) g(R(U, \xi) Z, \xi)+\eta(V) g(R(U, Y) Z, \xi) \\
- & g(Z, Y) g(R(U, V) \xi, \xi)+\eta(Z) g(R(U, V) Y, \xi)] .
\end{aligned}
$$

By the help of (3.8), (3.21) can be written as

$$
\begin{aligned}
& 0=\left[L_{R}+\left(\alpha^{2}+\xi(\alpha)\right)\right]\left[R(U, V, Z, Y)-\left(\alpha^{2}+\xi(\alpha)\right)[-g(Z, V) \eta(U) \eta(Y)\right. \\
&+ g(Z, U) \eta(V) \eta(Y)-g(Y, U) g(Z, V)+g(U, Y) \eta(V) \eta(Z) \\
&+g(V, Z) \eta(Y) \eta(U)-g(Y, Z) \eta(V) \eta(U)-g(V, Y) \eta(U) \eta(Z) \\
&+g(V, Y) g(Z, U)+g(Y, Z) \eta(U) \eta(V)-g(Z, U) \eta(V) \eta(Y) \\
&+g(Y, V) \eta(U) \eta(Z)-g(Y, U) \eta(Z) \eta(V)]] .
\end{aligned}
$$

Then the sum for $1 \leq i \leq 3$ of (3.22) for suitable contraction yields

$$
0=\left[L_{R}+\left(\alpha^{2}+\xi(\alpha)\right)\right]\left[S(V, Z)+2\left(\alpha^{2}+\xi(\alpha)\right) g(V, Z)\right] .
$$

Thus there are two cases with the help of (3.23). (3.23) just satisfies if either $-L_{R}=\left(\alpha^{2}+\xi(\alpha)\right)$ or $S(V, Z)=\lambda g(V, Z)$ where $\lambda=-2\left(\alpha^{2}+\xi(\alpha)\right)$. This completes the proof.

Theorem 3.4 Let $M^{3}$ be a pseudosymmetric alpha-Kenmotsu manifold. If the manifold $M^{3}$ has a non-zero constant function such that $L_{R} \neq-\alpha^{2}$ then the manifold $M^{3}$ is an Einstein manifold with $\lambda=-2 \alpha^{2}$ where $\alpha$ is a positive constant.

Proof According to the hypothesis, taking into consideration the above theorem we have

$$
0=\left[L_{R}+\alpha^{2}\right]\left[S(V, Z)+2 \alpha^{2} g(V, Z)\right]
$$

where $\alpha$ is a positive constant. Here, if we put $L_{R} \neq-\alpha^{2}$ then we obtain

$$
S(V, Z)=-2 \alpha^{2} g(V, Z) .
$$

This completes the proof. 


\section{Conclusions}

In this paper, we are especially interested in pseudosymmetric alpha-Kenmotsu manifolds. Some certain results are obtained related to curvature tensors on such manifolds. Our forthcoming paper is devoted to investigating alpha-Kenmotsu manifolds satisfying the other pseudosymmetry conditions. In particular, we study Ricci pseudosymmetry and generalized Ricci pseudosymmetry conditions. It is well known that open problems are so interesting in this area, especially for almost contact structures.

\section{Acknowledgments}

The author is grateful to the referee for valuable comments and suggestions.

\section{References}

1. Blair. D. E. (1977). Two remarks on contact metric structures. Tôhoku Mathematical Journal, 29: 319-324.

2. Calvaruso, G. and Perrone, D. (2001). Semi-symmetric contact metric three-manifolds. Yokohama Mathematical Journal, 49: 149-161.

3. Deszcz, R. (1992). On pseudosymmetric spaces. Bull. Belg. Math. Soc. Ser. A, 44: 1-34.

4. Dileo G. and Pastore M. (2007). Almost Kenmotsu manifolds and local symmetry. Bulletin of the Belgian Mathematical Society-Simon Stevin, 14: 343-354.

5. Hashimoto, N. and Sekizawa, M. (2000). Three dimensional conformally flat pseudo-symmetric spaces of constant type. Arch. Math. (Brno), 36: 279-286.

6. Janssens, D. and Vanhecke, L. (1981). Almost contact structures and curvature tensors.Kodai Mathematical Journal, 4: 1-27.

7. Jun, J., De, U.C and Pathak, G. (2005). On Kenmotsu manifolds. Journal of the Korean Math. Soc., 42: 435445.

8. Kenmotsu, K. (1972). A class of contact Riemannian manifold. Tôhoku Mathematical Journal, 24: 93-103.

9. Nomizu, K. (1968). On hypersurfaces satisfying a certain condition on the curvature tensor. Tôhoku Math. Journal, 20: 46-69.

10. Ogawa, Y. (1977). A condition for a compact Kaehlerian space to be locally symmetric. Natural Science Report. Ochanomizu University, 28: 21-23.

11. Özgür, C. (2006). On Kenmotsu manifolds satisfying certain pseudo symmetry conditions. World App. Sci. J., 1(2): 144-149.

12. Öztürk H., Aktan N. and Murathan C. (2010). On $\alpha$-Kenmotsu manifolds satisfyin certain conditions. Applied Sci., 12: 115-126.

13. Öztürk, H. (2017). On $\alpha$-Kenmotsu manifolds satisfying semi-symmetric conditions. Konuralp Journal of Mathematics, 5(2): 192-206.

14. Szabó, Z. I. (1982). Structure theorem on Riemannian spaces satisfying R.R=0 Journal of Diff. Geo., 17: 531-582. 
15. Venkatesha, K.T. and Divyashree, G. (2017). Three Dimensional f-Kenmotsu manifold satisfying certain curvature conditions. Cubo A Math. Journal, 19(1): 79-87.

16. Yano, K. and Kon, M. (1984). Structures on manifolds. Series in Pure Mathematics, 3.World Sci. Publ. Corp., Singapore. 